\title{
Incidence rate of prostate cancer in men treated for erectile dysfunction with phosphodiesterase type 5 inhibitors: retrospective analysis
}

\begin{abstract}
Anthony H Chavez ${ }^{1}$, K Scott Coffield ${ }^{1}$, M Hasan Rajab ${ }^{2,3}$ and Chanhee Jo ${ }^{2}$
The purpose of this study was to determine the incidence rate of prostate cancer among men with erectile dysfunction (ED) treated with phosphodiesterase type 5 inhibitors (PDE-5i) over a 7-year period vs. men with ED of the same age and with similar risk factors who were not treated with PDE-5i. In a retrospective review of electronic medical records and billing databases between the years 2000 and 2006, men with ED between the ages of 50 and 69 years and no history of prostate cancer prior to 2000 were identified. These individuals were divided into two groups: 2362 men who had treatment with PDE-5i, and 2612 men who did not have treatment. Demographic data in each group were compared. During the study period, 97 (4.1\%) men with ED treated with PDE-5i were diagnosed with prostate cancer compared with $258(9.9 \%)$ men with ED in the non-treated group $(P<00001)$. A higher percentage of African Americans were treated with PDE-5i vs. those who were not $(10.5 \%$ vs. $7.1 \% ; P<0.0001)$. The PDE-5i group had lower documented diagnosis of elevated prostate-specific antigen ( $10.0 \%$ vs. $13.1 \% ; P=0.0008)$ and higher percentage of benign prostatic hyperplasia (38.4\% vs. 35.1\%; $P=0.0149$ ). Men with ED treated with PDE-5i tended to have less chance (adjusted odds ratio: $0.4 ; 95 \%$ confidence intervals: $0.3-0.5 ; P<0.0001$ ) of having prostate cancer. Our data suggest that men with ED treated with PDE-5i tended to have less of a chance of being diagnosed with prostate cancer. Further research is warranted.
\end{abstract}

Asian Journal of Andrology (2013) 15, 246-248; doi:10.1038/aja.2012.162; published online 28 January 2013

Keywords: erectile dysfunction (ED); phosphodiesterase 5 inhibitors (PDE-5i); prostate cancer; prostatic neoplasms

\section{INTRODUCTION}

Several recent studies have suggested an association between sexual activity and risk of prostate cancer in older men. ${ }^{1,2}$ Some have proposed that increased sexual activity was an indicator of increased androgenic activity and thus a higher risk for prostate cancer. ${ }^{3}$ However, Leitzmann et al., ${ }^{2}$ in a large prospective study, found that high ejaculation frequency was related to decreased risk of total and organ-confined prostate cancer. One theory is that infrequent ejaculation leads to retained carcinogenic secretions in the prostatic acini. ${ }^{4}$

Erectile dysfunction (ED) is defined as 'the inability to attain and/or maintain penile erection sufficient for satisfactory sexual performance'. In patients with $\mathrm{ED}$, ejaculatory frequency is expected to be decreased. ${ }^{5}$ It would be valuable to assess if treating men with ED with phosphodiesterase type 5 inhibitors (PDE-5i) has any effect on the incidence of prostate cancer based on Leitzmann's findings. Our hypothesis was that the incidence rate of prostate cancer would be higher in the PDE-5i-treated group due to detection bias.

\section{MATERIALS AND METHODS}

This was an institutional review board-approved retrospective review of electronic medical records (EMR) and billing databases at our institution between the years 2000 and 2006. Using the international classification of disease (ICD9) code, we identified men between the ages of 50 and 69 diagnosed with ED with no history of prostate cancer prior to 2000. Age was determined at the date of enrollment. Study participants were divided into two groups: those who had received treatment with PDE-5i and those who had not. PDE-5i included tadalafil, vardenafil and sildenafil.

Prostate cancer was confirmed by prostate biopsy with histological diagnosis of prostate adenocarcinoma documented in the EMR. Demographics and baseline characteristics including age, ethnicity, benign prostatic hyperplasia $(\mathrm{BPH})$ and diagnosis of elevated prostate-specific antigen (PSA) $\left(>4.0 \mathrm{ng} \mathrm{dl}^{-1}\right)$ were compared between the two study groups. BPH was diagnosed by the physician documentation in the EMR, or by current procedural terminology code that the physician submitted for billing. As in actual practice, this was a clinical diagnosis, not based on pathology or specific objective criteria.

Inclusion criteria for the PDE-5i treatment group required the medication documented in all encounters over an one-year minimum period. Due to the limitations of the retrospective design, more strict criteria for determining inclusion criteria of the PDE-5i group were not feasible.

For categorical variables, a chi-square test was used to compare the patients with and without PDE-5i. Continuous variables were first ${ }_{1}^{1}$ Departments of Urology, Scott \& White Healthcare, Temple, Texas 76508, USA; ${ }^{2}$ Departments of Biostatistics, Scott \& White Healthcare, Temple, Texas 76508, USA and
${ }^{3}$ Deparment of Biostatistics, College of Medicine, Alfaisal University, Riyadh 11533, Saudi Arabia Correspondence: Dr KS Coffield (scoffield@sw.org)

Received: 30 March 2012; Revised: 6 September 2012; Accepted: 11 December 2012; Published online: 28 January 2013 
Table 1 General patient characteristics by PDE-5i treatment status

\begin{tabular}{|c|c|c|c|}
\hline & Treated with PDE-5i $(\mathrm{N}=2362)$ & Not treated with $P D E-5 i(\mathrm{~N}=2612)$ & $\mathrm{P}$ value \\
\hline Age (year, mean \pm s.d.) & $61.0 \pm 4.8$ & $61.4 \pm 4.9$ & 0.0044 \\
\hline Race $(n, \%)$ & & & 0.0002 \\
\hline Caucasian & $1608(68.1)$ & $1820(69.7)$ & \\
\hline African American* & $249(10.5)$ & $186(7.1)$ & $<0.0001$ \\
\hline Hispanic & $157(6.6)$ & $172(6.6)$ & \\
\hline Others & $348(14.7)$ & $434(16.6)$ & \\
\hline $\mathrm{BPH}(n, \%)$ & $907(38.4)$ & $916(35.1)$ & 0.0149 \\
\hline Elevated PSA ( $n, \%)$ & 237 (10.0) & $342(13.1)$ & 0.0008 \\
\hline Prostate cancer $(n, \%)$ & $97(4.1)$ & $258(9.9)$ & $<0.0001$ \\
\hline
\end{tabular}

Abbreviations: BPH, benign prostatic hyperplasia; PDE-5i, phosphodiesterase type 5 inhibitors; PSA, prostate-specific antigen.

* P value for African American's only.

examined for normality and then compared using two-sample $t$-test for independent samples or Wilcoxon non-parametric two-sample test, as appropriate. We used multiple logistic regression in the analysis of the study, having prostate cancer between 2000 and 2006. A $P$ value of less than 0.05 indicated a statistical significance and SAS version 9.2 (SAS Institute Inc., Cary, NC, USA) was used for the statistical analysis.

\section{RESULTS}

A total of 4974 men were included in the study, $2362(47.5 \%)$ of whom had a record of being prescribed a PDE-5i. Mean age was $61.2 \pm 4.85$ years, ranging from 50 to 69 years. Sixty-nine percent were Caucasians, $8.8 \%$ were Africa Americans, 6.6\% were Hispanic and $15.7 \%$ were classified as others. Mean ages for the treated (PDE-5i) and non-treated groups were $61.0 \pm 4.8 v s .61 .4 \pm 4.9$ years, respectively $(P=0.0044)$. There was a higher percentage of African Americans in the treated (PDE-5i) group $(10.5 \%$ vs. $7.1 \% ; P<0.0001)$ (Table 1).

Fewer patients in the PDE-5i group had a documented diagnosis of elevated PSA ( $10.0 \%$ vs. $13.1 \% ; P=0.0008)$. Prostate biopsies were performed in patients with elevated PSA and abnormal digital prostate exams. There was a higher percentage of patients with BPH in the PDE-5i treatment group (38.4\% vs. $35.1 \% ; P=0.0149)$. Out of the 2362 men in the treated (PDE-5i) group, $97(4.1 \%)$ had a record of prostate cancer $v$ s. $258(9.9 \%)$ out of the 2612 in the non-treated group $(P<0.0001)$. In the multiple logistic regression analysis, use of PDE-5i, age and elevated PSA were significant predictors of prostate cancer (Table 2). On the other hand, race and BPH were not significant predictors of prostate cancer though $\mathrm{BPH}$ was on the borderline $(P=0.0692)$ (Table 3).

Men with ED who were treated with PDE-5i tended to have less chance (adjusted odds ratio: $0.4 ; 95 \%$ confidence intervals: $0.3-0.5$; $P<0.0001$ ) of having prostate cancer. Treatment univariate and multiple logistic regression analyses are summarized in Tables 1-3, respectively.

Table 2 Logistic regression analysis on incidence of prostate cancer (without BPH)

\begin{tabular}{lccc}
\hline & Adjusted odds ratio & 95\% confidence intervals & P value \\
\hline PDE-5i treatment & 0.4 & $0.3-0.5$ & $<0.0001$ \\
Age & 1.1 & $1.0-1.1$ & $<0.0001$ \\
Elevated PSA & 21.6 & $16.8-37.8$ & $<0.0001$ \\
\hline
\end{tabular}

Abbreviations: $\mathrm{BPH}$, benign prostatic hyperplasia; PDE-5i, phosphodiesterase type 5 inhibitors; PSA, prostate-specific antigen.

\section{DISCUSSION}

In this retrospective study among men with $\mathrm{ED}$, the use of PDE-5i was associated with a decreased incidence rate of prostate cancer. The use of PDE-5i, PSA and advanced age were significant predictors of prostate cancer. Interestingly, there were more African-American men in the PDE-5i treatment group, predisposing these patients to an increased risk of prostate cancer. The number of patient prostate biopsies varied with PSA elevation and abnormal digital prostate exam. We were unable from this database to determine whether the patients in the treatment group who had BPH had more severe symptoms of BPH prior to PDE-5i treatment.

It is clear from the more recent literature that $\mathrm{BPH}$ is common and that the PDE-5i treatment has been acknowledged as an acceptable alternative in this frequently diagnosed disease. ${ }^{6}$ There is no preexisting data to suggest that PDE-5i treatment increases BPH risk and these data do not suggest that. Although $\mathrm{Krain}^{3}$ proposed that increased sexual activity was an indicator of increased androgenic activity and thus a higher risk for prostate cancer, our results showed that the risk for prostate cancer decreased in those who used PDE-5i.

Several theories may explain the potential association between PDE-5i and decreased risk of prostate cancer. Patients with ED treated with PDE-5i are expected to have a higher ejaculation frequency than those who are untreated. Leitzmann et al.'s large prospective cohort study ${ }^{2}$ demonstrated a protective effect of increased ejaculation frequency with regard to prostate cancer diagnosis. Furthermore, there may be a protective effect from the physiological and molecular activity of the PDE-5i. Zenzmaier et al. ${ }^{7}$ studied the in vitro effects of PDE$5 \mathrm{i}$ on prostate cells. They reported that tadalafil at $2.5 \mu \mathrm{moll}^{-1}$ had pronounced effect on proliferation of prostate stromal cells though required $25 \mu \mathrm{moll}^{-1}$ concentration that notably reduced proliferation of prostate epithelial cells. ${ }^{7}$ Zenzmaier et al. ${ }^{7}$ confirmed that PDE-5 was highly expressed in the stromal compartment of the prostate but not the epithelium. Hypoxia is common in prostate tumors, promoting

Table 3 Logistic regression analysis on incidence of prostate cancer (with BPH in the model)

\begin{tabular}{lccr}
\hline & Adjusted odds ratio & 95\% confidence intervals & P value \\
\hline PDE-5i treatment & 0.4 & $0.3-0.5$ & $<0.0001$ \\
Age & 1.1 & $1.0-1.1$ & $<0.0001$ \\
BPH & 0.8 & $0.6-1.0$ & 0.0692 \\
Elevated PSA & 23.7 & $18.0-31.3$ & $<0.0001$ \\
\hline
\end{tabular}

Abbreviations: BPH, benign prostatic hyperplasia; PDE-5i, phosphodiesterase type 5 inhibitors; PSA, prostate-specific antigen. 
tumor progression. ${ }^{8}$ There is evidence that hypoxia plays a pivotal role in cancer adaptive responses leading to poor clinical outcomes for patients with prostate cancer. ${ }^{9}$ Khandrika et al. ${ }^{10}$ recently presented data demonstrating the effects of intermittent hypoxia on prostate cancer. These data suggest that intermittent hypoxia-reoxygenation promotes an aggressive phenotype of prostate cancer via hypoxia-induced increased androgen receptor activity, increased androgen receptor responsive promoter activity independent of androgens and increased hypoxia-inducible factor- $1 \alpha$ levels and activity. ${ }^{10}$ It is well known that PDE-5i facilitate circulatory perfusion and reduce hypoxia by decreasing the degradation of cyclic guanosine monophosphate. ${ }^{11} \mathrm{PDE}-5 \mathrm{i}$ may alter the development and progression of prostate cancer by decreasing intermittent hypoxia-reoxygenation effect and prevention of the formation of a tumor-reactive stroma.

Hamilton et al. ${ }^{12}$ reported the first study to characterize cGMP- and cAMP-dependent PDE activity in prostate cancer cell lines. Flow cytometry was used to determine the effects of PDE inhibition on surface major histocompatibility complex class I-related chain A, a natural killer cell-activating ligand in a mouse model to evaluate the in vivo effects of PDE inhibition on the growth of human prostate cancer cells. ${ }^{12}$ PDE (PDE5 and PDE11) inhibition completely reversed hypoxia-induced shedding of the immune-stimulatory molecule, major histocompatibility complex class I-related chain A, and attenuated the growth of human prostate tumor xenografts ( $\mathrm{Du}-145$ cells) in a natural killer cell-competent murine model.

The progression of prostate cancer is associated with malignant cellular phenotype characterized by increased tumor cell invasion, metastasis and escape from immune destruction. The acquisition of these malignant traits is influenced by genetic and epigenetic factors, but also by microenvironmental stressors such as hypoxia. ${ }^{12}$ Though the relationship between PDE-5i therapy and development and progression of prostate cancer remains to be determined, the use of PDE-5i therapy may play a role in this commonly occurring cancer.

To our knowledge, this is the first report seeking an association between prostate cancer and PDE-5i therapy. This study includes the limitations associated with its single-center location, retrospective design, potential variation in physician documentation in the EMR, lack of strict criteria for prescribed PDE-5i medication and lack of family history documentation. The limitation of subjects with varying lengths of exposure to PDE-5i therapy, because of entering the study at different times during the 7-year period of data collection, is acknowledged. This limitation and others are best addressed by a prospective trial. However, data from this study suggest that an association between the rate of incidence of prostate cancer and PDE-5i may exist.

\section{CONCLUSION}

Our data suggest that men with ED who were treated with PDE-5i had a reduced risk of being diagnosed with prostate cancer. We believe that further research is justified.

\section{AUTHOR CONTRIBUTIONS}

AHC conceived the study, participated in the study design and drafted the manuscript. KSC coconceived the study, participated in the study design and revised the manuscript. MHR participated in the study design, revised the manuscript and performed statistical analysis. CJ reviewed the statistical analysis of the data. All authors read and approved the final manuscript.

\section{COMPETING FINANCIAL INTERESTS}

All authors declare that there are no competing financial interests.

\section{ACKNOWLEDGMENTS}

We would like to thank Anne M. Robertson for her help in data collection, and Gina Du Par for her editorial assistance.

1 Giles GG, Severi G, English DR, McCredie MR, Borland R et al. Sexual factors and prostate cancer. BJU Int 2003; 92: 211-6.

2 Leitzmann MF, Platz EA, Stampfer MJ, Willett WC, Giovannucci E. Ejaculation frequency and subsequent risk of prostate cancer. JAMA 2004; 291: 1578-86.

3 Krain LS. Some epidemiologic variables in prostatic carcinoma in California. Prev Med 1974; 3: 154-9.

4 Isaacs JT. Prostatic structure and function in relation to the etiology of prostate cancer. Prostate 1983; 4: 351-66.

5 Steidle CP, McCullough AR, Kaminetsky JC, Crowley AR, Siegel RL et al. Early Sildenafil dose optimization and personalized instruction improves the frequency, flexibility, and success of sexual intercourse in men with erectile dysfunction. Int $J$ Impot Res 2007; 19: 154-60.

6 Egerdie RB, Auerbach S, Roehrborn CG, Costa P, Garza MS et al. Tadalafil 2.5 or $5 \mathrm{mg}$ administered once daily for 12 weeks in men with both erectile dysfunction and signs and symptoms of benign prostatic hyperplasia: results of a randomized, placebocontrolled, double-blind study. J Sex Med 2012; 9: 271-81.

7 Zenzmaier C, Sampson N, Pernkopf D, Plas E, Untergasser G et al. Attenuated proliferation and trans-differentiation of prostatic stromal cells indicate suitability of phosphodiesterase type 5 inhibitors for prevention and treatment of benign prostatic hyperplasia. Endocrinology 2010; 151: 3975-84.

8 Rudolfsson SH, Bergh A. Hypoxia drives prostate tumour progression and impairs the effectiveness of therapy, but can also promote cell death and serve as a therapeutic target. Expert Opin Ther Targets 2009; 13: 219-25.

9 Movsas B, Chapman JD, Greenberg RE, Hanlon AL, Horwitz EM et al. Increasing levels of hypoxia in prostate carcinoma correlate significantly with increasing clinical stage and patient age: an Eppendorf pO(2) study. Cancer 2000; 89: 2018-24.

10 Khandrika L, Lieberman R, Koul S, Kumar B, Meacham R et al. Hypoxia-reoxygenation promotes aggressive phenotype in prostate cancer cells. (Abstract) J Urol 2008; 179 (Suppl): 192.

11 Corbin JD, Francis SH. Molecular biology and pharmacology of PDE-5-inhibitor therapy for erectile dysfunction. J Androl 2003; 24: 538-41.

12 Hamilton TK, Hu N, Kolomitro K, Bell EN, Maurice DH et al. Potential therapeutic applications of phosphodiesterase inhibition in prostate cancer. World J Urol; e-pub ahead of print 2 March 2012; doi:10.1007/s00345-012-0848-7. 